\title{
Classical Bianchi Type I Cosmology in K-Essence Theory
}

\author{
J. Socorro, ${ }^{1}$ Luis O. Pimentel, ${ }^{2}$ and Abraham Espinoza-García ${ }^{1}$ \\ ${ }^{1}$ Departamento de Física, DCeI, Universidad de Guanajuato, Campus León, Apartado Postal E-143, 37150 León, GTO, Mexico \\ ${ }^{2}$ Departamento de Física, Universidad Autónoma Metropolitana, Apartado Postal 55-534, 09340 México, DF, Mexico
}

Correspondence should be addressed to J. Socorro; socorro@fisica.ugto.mx

Received 23 June 2014; Revised 8 September 2014; Accepted 9 September 2014; Published 14 October 2014

Academic Editor: Rong-Gen Cai

Copyright (C) $2014 \mathrm{~J}$. Socorro et al. This is an open access article distributed under the Creative Commons Attribution License, which permits unrestricted use, distribution, and reproduction in any medium, provided the original work is properly cited. The publication of this article was funded by SCOAP ${ }^{3}$.

We use one of the simplest forms of the K-essence theory and we apply it to the classical anisotropic Bianchi type I cosmological model, with a barotropic perfect fluid $(p=\gamma \rho)$ modeling the usual matter content and with cosmological constant $\Lambda$. Classical exact solutions for any $\gamma \neq 1$ and $\Lambda=0$ are found in closed form, whereas solutions for $\Lambda \neq 0$ are found for particular values in the barotropic parameter. We present the possible isotropization of the cosmological model Bianchi I using the ratio between the anisotropic parameters and the volume of the universe. We also include a qualitative analysis of the analog of the Friedmann equation.

\section{Introduction}

In recent times, some attempts to unify the description of dark matter, dark energy, and inflation, by means of a scalar field with nonstandard kinetic term, have been conducted [16]. The K-essence theory is based on the idea of a dynamical attractor solution which causes it to act as a cosmological constant only at the onset of matter domination. Consequently, $\mathrm{K}$-essence overtakes the matter density and induces cosmic acceleration at about the present epoch. Usually K-essence models are restricted to the Lagrangian density of the form $[4,7-10]$

$$
S=\int d^{4} x \sqrt{-g}[f(\phi) \mathscr{G}(X) 5-V(\phi)],
$$

where the canonical kinetic energy is given by $\mathscr{G}(X)=X=$ $-(1 / 2) \nabla_{\mu} \phi \nabla^{\mu} \phi$. K-essence was originally proposed as a model for inflation and then as a model for dark energy, along with explorations of unifying dark energy and dark matter $[7,11,12]$. Other motivations to consider the action above originate from string theory $[13,14]$. For more details for Kessence applied to dark energy you can see [15] and references therein.

In this framework, gravitational and matter variables have been reduced to a finite number of degrees of freedom. For homogenous cosmological models the metric depends only on time and gives a model with a finite dimensional configuration space, called minisuperspace. In this work, we use this formulation to obtain classical solutions to the anisotropic Bianchi type I cosmological model with a perfect fluid. This class of models was considered initially in this formalism by Chimento and Forte [16]. The first step is to write the theory for the Bianchi type I model in the usual manner; that is, we calculate the corresponding energy-momentum tensor to the scalar field and give the equivalent Lagrangian density. Next, by means of a Legendre transformation, we proceed to obtain the canonical Lagrangian $\mathscr{L}_{\text {can }}$, from which the classical Hamiltonian $\mathscr{H}$ can be found.

One of the simplest K-essence models, without selfinteraction, has the following Lagrangian density:

$$
\mathscr{L}_{\text {geo }}=R+f(\phi) \mathscr{G}(X),
$$

where $R$ is the scalar curvature and $f(\phi)$ is an arbitrary function of the scalar field.

From the Lagrangian (2) we can build the complete action:

$$
I=\int_{\Sigma} \sqrt{-g}\left(\mathscr{L}_{\text {geo }}+\mathscr{L}_{\Lambda}+\mathscr{L}_{\text {mat }}\right) d^{4} x
$$


where $\mathscr{L}_{\text {mat }}$ is the matter Lagrangian, $\mathscr{L}_{\Lambda}=2 \Lambda$ is the cosmological constant Lagrangian, and $g$ is the determinant of the metric tensor. The field equations for this theory are

$$
\begin{gathered}
G_{\alpha \beta}+\Lambda g_{\alpha \beta}+f(\phi)\left[\mathscr{G}_{X} \phi_{, \alpha} \phi_{, \beta}+\mathscr{G}_{\alpha \beta}\right]=-T_{\alpha \beta}, \\
f(\phi)\left[\mathscr{G}_{X} \phi_{; \beta}^{, \beta}+\mathscr{G}_{X X} X_{; \beta} \phi^{, \beta}\right]+\frac{d f}{d \phi}\left[\mathscr{G}-2 X \mathscr{G}_{X}\right]=0,
\end{gathered}
$$

where we work in units with $8 \pi G=1$ and, as usual, the semicolon means a covariant derivative and a subscripted $X$ denotes differentiation with respect to $X$.

The same set of (4a), (4b) is obtained if we consider the scalar field $X(\phi)$ as part of the matter content; that is to say, $\mathscr{L}_{X, \phi}=f(\phi) \mathscr{G}(X)$ with the corresponding energymomentum tensor:

$$
\mathscr{T}_{\alpha \beta}=f(\phi)\left[\mathscr{G}_{X} \phi_{, \alpha} \phi_{, \beta}+\mathscr{G}(X) g_{\alpha \beta}\right] .
$$

Considering the energy-momentum tensor of a barotropic perfect fluid,

$$
T_{\alpha \beta}=(\rho+P) u_{\alpha} u_{\beta}+P g_{\alpha \beta},
$$

where $u_{\alpha}$ is the four-velocity, which satisfies the relation $u_{\mu} u^{\mu}=-1, \rho$ is the energy density, and $P$ is the pressure of the fluid. For simplicity we consider a comoving perfect fluid. The pressure, the energy density, and the four-velocity, corresponding to the energy-momentum tensor of the field $X$, become

$$
\begin{gathered}
P(X)=f(\phi) \mathscr{G}, \quad \rho(X)=f(\phi)\left[2 X \mathscr{G}_{X}-\mathscr{G}\right], \\
u_{\mu}=\frac{\nabla_{u} \phi}{\sqrt{2 X}} ;
\end{gathered}
$$

thus, the barotropic parameter is

$$
\omega_{X}=\frac{\mathscr{G}}{2 X \mathscr{G}_{X}-\mathscr{G}}
$$

and we notice that the case of a constant barotropic index $\omega_{X}$ (with the exception $\omega_{X}=0$ ) can be obtained by the $\mathscr{G}$ function

$$
\mathscr{G}=X^{\left(1+\omega_{X}\right) / 2 \omega_{X}} .
$$

We have the following states in the evolution of our universe in this formalism:

$$
\begin{aligned}
\text { stiff matter: } \omega_{X}=1, & \longrightarrow \mathscr{G}(X)=X, \\
\text { Radiation: } \omega_{X}=\frac{1}{3}, & \longrightarrow \mathscr{G}(X)=X^{2}, \\
\text { inflation like: } \omega_{X}=-\frac{1}{3}, & \longrightarrow \mathscr{G}(X)=\frac{1}{X}, \\
\omega_{X}=-\frac{2}{3}, & \longrightarrow \mathscr{G}(X)=\frac{1}{\sqrt[4]{X}} .
\end{aligned}
$$

The mathematical analysis for the last two cases is very complicated in both regimes, classical and quantum one.
For quantum radiation case, the resulting Wheeler-DeWitt equation appears as fractionary differential equation, and the results will be reported elsewhere. In [6], the authors present the analysis to radiation era using dynamical systems obtaining bouncing solutions. It is worthy to mention that (4b) was especialized in [17] to flat FRW cosmological model and solved using an extended tachyon field from which an extended Chaplygin gas can arise, which interpolates between a power law phase and a de Sitter phase. The author found the general solutions for linear expansion to the functional $G(X)$ and particular $f(\phi) \rightarrow \phi^{-2}$ and showed from the kinematic point of view that this model and the quintessence scalar field one driven by an exponential potential are the same, but they are dynamically nonequivalent because the $\mathrm{K}$-field and the scalar potential are linked by the Einstein equation, similar to our next equations (19). However our results contain other functions $f(\phi)$; then we argued that explanation to the corresponding observational data and the kinematic properties, since each formalism will have big differences.

1.1. Anisotropic Cosmological Bianchi Class A Models, $f(\phi)=$ Constant. Considering the cosmological anisotropic Bianchi class A models with metric (23), (4b) in terms of $X$ becomes (here and all where appear the ' means, ${ }^{\prime}=d / d \tau=d / N d t$, with $t$ the usual cosmic time)

$$
\left[\mathscr{G}_{X}+2 X \mathscr{G}_{X X}\right] X^{\prime}+6 \Omega^{\prime} X \mathscr{G}_{X}=0,
$$

and its corresponding solution becomes

$$
X \mathscr{G}_{X}^{2}=\eta e^{-6 \Omega},
$$

with $\eta$ a constant.

Note that (12) gives us the possible solution $X(A)$, as a function of the scale factor, and therefore the behavior of all physical properties of the K-essence (like $\rho, P$ ) is completely determined by the function $X$ and does not depend on the evolution of the other types of energy density. The only dependence of the K-essence component on other components enters through $A(\tau)=e^{\Omega(\tau)}$ in Bianchi class $\mathrm{A}$ cosmological models.

In the following we present the analysis when $f(\phi)$ is a constant and generic function of the field $\phi$ assuming a Bianchi type I metric, which is the anisotropic generalization of flat FRW cosmological model, and we present the solution in quadrature form.

1.1.1. Quintessence Radiation Like Case: $\mathscr{G}=X^{2}$ and $f(\phi)=$ Constant. Using the equation

$$
X \mathscr{G}_{X}^{2}=\eta e^{-6 \Omega}
$$

for the energy kinetic we have the form

$$
X=\sqrt[3]{\frac{\eta}{4}} e^{-2 \Omega},
$$

then the field $\phi$ has the solution

$$
\Delta \phi=\sqrt{2} \sqrt[6]{\frac{\eta}{4}} \int e^{-\Omega} d \tau .
$$


1.1.2. Quintessence Stiff Fluid Like Case: $\mathscr{G}=X$ and $f(\phi) \neq$ Constant. The field equations for this particular case are

$$
\begin{gathered}
G_{\alpha \beta}+\Lambda g_{\alpha \beta}+f(\phi)\left(\phi_{, \alpha} \phi_{, \beta}-\frac{1}{2} g_{\alpha \beta} \phi_{, \gamma} \phi^{, \gamma}\right)=-T_{\alpha \beta}, \\
2 f(\phi) \phi_{; \alpha}^{, \alpha}+\frac{d f}{d \phi} \phi_{, \gamma} \phi^{, \gamma}=0
\end{gathered}
$$

and the energy-momentum tensor (5) has the following form:

$$
\mathscr{T}_{\alpha \beta}=f(\phi)\left(\phi_{, \alpha} \phi_{, \beta}-\frac{1}{2} g_{\alpha \beta} \phi_{, \gamma} \phi^{\gamma}\right)
$$

In this new line of reasoning, action (3) can be rewritten as a geometrical part and matter content (usual matter plus a term that corresponds to the exotic scalar field component of the K-essence theory).

The equation of motion for the field $\phi(16 b)$ has the following property, using the metric of the Bianchi type I model (however, this is satisfied by all cosmological Bianchi class A models):

$$
3 \Omega^{\prime} \phi^{\prime} f+\phi^{\prime \prime} f+\frac{1}{2} \frac{d f}{d \phi} \phi^{\prime 2}=0,
$$

which can be integrated at once with the following result:

$$
\frac{1}{2} f(\phi) \phi^{\prime 2}=\eta e^{-6 \Omega}, \quad \longrightarrow \int \sqrt{f(\phi)} d \phi=\sqrt{2 \eta} \int e^{-3 \Omega(\tau)} d \tau,
$$

where $\eta$ is an integration constant and has the same sign as $f(\phi)$. Considering the particular form of $f(\phi)=\omega \phi^{m}$ or $f(\phi)=\omega e^{m \phi}$ with $m$ and $\omega$ being constants, the classical solutions for the field $\phi$ in quadrature are

$$
\phi(\tau)=\left\{\begin{array}{c}
{\left[(m+2) \sqrt{\frac{\eta}{2 \omega}} \int e^{-3 \Omega} d \tau\right]^{2 /(m+2)},} \\
f(\phi)=\omega \phi^{m}, \quad m \neq-2, \\
\operatorname{Exp}\left\{\sqrt{\frac{2 \eta}{\omega}} \int e^{-3 \Omega} d \tau\right\}, \\
f(\phi)=\omega \phi^{-2}, \quad m=-2, \\
\frac{2}{m} \ln \left[m \sqrt{\frac{\eta}{2 \omega}} \int e^{-3 \Omega} d \tau\right], \\
f(\phi)=\omega e^{m \phi}, \quad m \neq 0, \\
\sqrt{2 \eta} \int e^{-3 \Omega} d \tau \quad m=0 .
\end{array}\right.
$$

In the particular gauge $N=24 e^{3 \Omega},(20)$ is simplified to (remember that $d \tau=N(t) d t$ )

$$
\phi(t)=\left\{\begin{array}{c}
{\left[24(m+2) \sqrt{\frac{\eta}{2 \omega}} t\right]^{2 /(m+2)},} \\
f(\phi)=\omega \phi^{m}, \quad m \neq-2, \\
\operatorname{Exp}\left\{24 \sqrt{\frac{2 \eta}{\omega}} t\right\}, \\
f(\phi)=\omega \phi^{-2}, \quad m=-2, \\
\frac{2}{m} \ln \left[24 m \sqrt{\frac{\eta}{2 \omega}} t\right], \\
f(\phi)=\omega e^{m \phi}, \quad m \neq 0, \\
24 \sqrt{2 \eta t}, \quad m=0 .
\end{array}\right.
$$

The complete solution to the quintessence scalar field $\phi$ depends strongly on the behavior of the scale factor of the cosmological model under consideration and particular form to the function $f(\phi)$; Bianchi type model appears mimetic in the $\Omega$ function, as in (15) and (19), except when one chooses a particular gauge which includes this function, as in (21).

In our particular case, it is evident that the contribution of the scalar field is equivalent to a stiff fluid with a barotropic equation of state $\gamma=1$. This is an instance of the results of the analysis of the energy-momentum tensor of a scalar field (17) by Madsen [18] for general relativity with scalar matter and by Pimentel [19] for the general scalar tensor theory. In both works a free scalar field is equivalent to a stiff matter fluid. In this way, we write action (3) in the usual form

$$
I=\int_{\Sigma} \sqrt{-g}\left(\frac{R}{2}+\mathscr{L}_{\Lambda}+\mathscr{L}_{\text {mat }}+\mathscr{L}_{\phi}\right) d^{4} x,
$$

and, consequently, the classical equivalence between the two theories. We can infer that this correspondence is also satisfied in the quantum regime, so we can use this structure for the quantization program, where the ADM formalism is well known for different classes of matter [20].

This work is arranged as follows. In Section 2 we construct the Lagrangian and Hamiltonian densities for the anisotropic Bianchi type I cosmological model. In Section 3 we present some ideas as the anisotropic cosmological model can obtain its isotropization via the mean volume function and next we obtain the classical exact solution for all values in the gamma parameter. Finally, Section 3 is devoted to some final remarks.

\section{Hamiltonian for the Bianchi Type I Cosmological Model}

Let us recall here the canonical formulation in the ADM formalism of the diagonal Bianchi class A models. The metric has the form

$$
\begin{aligned}
d s^{2} & =-(N d t)^{2}+e^{2 \Omega(t)}\left(e^{2 \beta(t)}\right)_{i j} \omega^{i} \omega^{j} \\
& =-d \tau^{2}+e^{2 \Omega(t)}\left(e^{2 \beta(t)}\right)_{i j} \omega^{i} \omega^{j},
\end{aligned}
$$


where $\Omega(t)$ is a scalar, $N$ is the lapse function, $\beta_{i j}(t)$ is a $3 \times 3$ diagonal matrix, $\beta_{i j}=\operatorname{diag}\left(\beta_{+}+\sqrt{3} \beta_{-}, \beta_{+}-\sqrt{3} \beta_{-},-2 \beta_{+}\right)$, $\omega^{i}$ are one-forms that characterize each cosmological Bianchi type model and obey $d \omega^{i}={ }_{\overline{2}} C_{j k}^{i} \omega^{j} \wedge \omega^{k}$, and $C_{j k}^{i}$ are the structure constants of the corresponding invariance group. For the Bianchi type I model we have

$$
\omega^{1}=d x^{1}, \quad \omega^{2}=d x^{2}, \quad \omega^{3}=d x^{3} .
$$

The total Lagrangian density then for this metric becomes

$$
\mathscr{L}_{\mathrm{I}}=e^{3 \Omega}\left[6 \frac{\dot{\Omega}^{2}}{N}-6 \frac{\dot{\beta}_{+}^{2}}{N}-6 \frac{\dot{\beta}_{-}^{2}}{N}+\frac{f(\phi)}{2 N} \dot{\phi}^{2}+2 N \rho+2 N \Lambda\right],
$$

using the standard definition of the momenta, $\Pi_{q^{\mu}}=$ $\partial \mathscr{L} / \partial \dot{q}^{\mu}$, where $q^{\mu}=\left(\Omega, \beta_{+}, \beta_{-}, \phi\right)$, we obtain

$$
\begin{array}{cc}
\Pi_{\Omega}=\frac{12}{N} e^{3 \Omega} \dot{\Omega}, & \longrightarrow \dot{\Omega}=\frac{N}{12} e^{-3 \Omega} \Pi_{\Omega}, \\
\Pi_{+}=-\frac{12}{N} e^{3 \Omega} \dot{\beta}_{+}, & \longrightarrow \dot{\beta}_{+}=-\frac{N}{12} e^{-3 \Omega} \Pi_{+}, \\
\Pi_{-}=-\frac{12}{N} e^{3 \Omega} \dot{\beta}_{-}, & \longrightarrow \dot{\beta}_{-}=-\frac{N}{12} e^{-3 \Omega} \Pi_{+}, \\
\Pi_{\phi}=\frac{f}{N} e^{3 \Omega} \dot{\phi}, & \longrightarrow \dot{\phi}=\frac{N}{f} e^{-3 \Omega} \Pi_{\phi},
\end{array}
$$

and, introducing them into the Lagrangian density, we obtain the canonical Lagrangian as $\mathscr{L}_{\text {canonical }}=\Pi_{q^{\mu}} \dot{q}^{\mu}-N \mathscr{H}$,

$$
\begin{aligned}
& \mathscr{L}_{\text {canonical }} \\
& \qquad \begin{aligned}
=\Pi_{q^{\mu}} \dot{q}^{\mu}-\frac{N}{24} e^{-3 \Omega}\{ & \Pi_{\Omega}^{2}+\frac{12}{f(\phi)} \Pi_{\phi}^{2}-\Pi_{+}^{2}-\Pi_{-}^{2} \\
& \left.-48 \mu_{\gamma} e^{-3(\gamma-1) \Omega}-48 \Lambda e^{6 \Omega}\right\},
\end{aligned}
\end{aligned}
$$

where we have used energy-momentum conservation, the law for a perfect fluid; $T_{; \nu}^{\mu \nu}=0, \rightarrow \rho=\mu_{\gamma} e^{-3(\gamma+1) \Omega}$; we assumed an equation of state $p=\gamma \rho$, so the corresponding Hamiltonian density is

$$
\begin{aligned}
\mathscr{H}_{\perp}=\frac{e^{-3 \Omega}}{24}( & -\Pi_{\Omega}^{2}-\frac{12}{f(\phi)} \Pi_{\phi}^{2}+\Pi_{+}^{2} \\
& \left.+\Pi_{-}^{2}+b_{\gamma} e^{-3(\gamma-1) \Omega}+48 \Lambda e^{6 \Omega}\right),
\end{aligned}
$$

with $b_{\gamma}=48 \mu_{\gamma}$.
2.1. Classical Equations. The corresponding Einstein field equations (16a) and (16b) for the anisotropic cosmological model Bianchi type I are the following (remember that the prime $^{\prime}$ is the derivative over the time $d \tau=N d t$ ):

$$
\begin{gathered}
3 \Omega^{\prime 2}-3 \beta_{+}^{\prime 2}-3 \beta_{-}^{\prime 2}-\frac{f}{4} \phi^{\prime 2}-\rho-\Lambda=0,2 \Omega^{\prime \prime} \\
+3 \Omega^{\prime 2}-3 \Omega^{\prime} \beta_{+}^{\prime}-3 \sqrt{3} \Omega^{\prime} \beta_{-}^{\prime}-\beta_{+}^{\prime \prime}+3 \beta_{+}^{\prime 2} \\
-\sqrt{3} \beta_{-}^{\prime \prime}+3 \beta_{-}^{\prime 2}+\frac{f}{4} \phi^{\prime 2}+p-\Lambda=0,2 \Omega^{\prime \prime} \\
+3 \Omega^{\prime 2}-3 \Omega^{\prime} \beta_{+}^{\prime}+3 \sqrt{3} \Omega^{\prime} \beta_{-}^{\prime}-\beta_{+}^{\prime \prime}+3 \beta_{+}^{\prime 2} \\
+\sqrt{3} \beta_{-}^{\prime \prime}+3 \beta_{-}^{\prime 2}+\frac{f}{4} \phi^{\prime 2}+p-\Lambda=0,2 \Omega^{\prime \prime} \\
+3 \Omega^{\prime 2}+6 \Omega^{\prime} \beta_{+}^{\prime}+2 \beta_{+}^{\prime \prime}+3 \beta_{+}^{\prime 2}+3 \beta_{-}^{\prime 2} \\
+\frac{f}{4} \phi^{\prime 2}+p-\Lambda=0, \\
\quad f\left(3 \Omega^{\prime} \phi^{\prime}+\phi^{\prime \prime}\right)+\frac{1}{2} \frac{d f}{d \phi} \phi^{\prime 2}=0 .
\end{gathered}
$$

The solution of this last equation was putted in (19),

$$
\frac{1}{2} f(\phi) \phi^{\prime 2}=\eta e^{-6 \Omega}, \quad \longrightarrow \int \sqrt{f(\phi)} d \phi=\sqrt{2 \eta} \int e^{-3 \Omega(\tau)} d \tau .
$$

The combination between the second and third equations gives us the solution for the anisotropic function $\beta_{-}$; also the sum of third and fourth equations, putting the $\beta_{-}$solution, gives us the form of the $\beta_{+}$function,

$$
\beta_{ \pm}(\tau)=a_{ \pm} \int e^{-3 \Omega(\tau)} d \tau,
$$

where $a_{ \pm}$are integration constants. So, (29) are rewritten as

$$
\begin{gathered}
3 \Omega^{\prime 2}=3 c_{1} e^{-6 \Omega}+\mu_{\gamma} e^{-3(\gamma+1) \Omega}+\Lambda, \quad c_{1}=a_{+}^{2}+a_{-}^{2}+\frac{\eta}{6}, \\
2 \Omega^{\prime \prime}+3 \Omega^{\prime 2}+3 \beta_{+}^{\prime 2}+3 \beta_{-}^{\prime 2}+\frac{f}{4} \phi^{\prime 2}+p-\Lambda=0 .
\end{gathered}
$$

2.2. Isotropization. The current observations of the cosmic background radiation set a very stringent limit to the anisotropy of the universe [21]; therefore it is important to consider the anisotropy of the solutions. Recalling the Friedmann equation (constraint equation),

$$
3 \Omega^{\prime 2}-3 \beta_{+}^{\prime 2}-3 \beta_{-}^{\prime 2}-\frac{f}{4} \phi^{\prime 2}-\rho-\Lambda=0,
$$

we can see that isotropization is achieved when the terms with $\beta_{ \pm}^{\prime 2}$ go to zero or are negligible with respect to the other terms in the differential equation. We find in the literature the criteria for isotropization, among others, $\left(\beta_{+}^{\prime 2}+\beta_{-}^{\prime 2}\right) / H^{2} \rightarrow 0$, $\left(\beta_{+}^{\prime 2}+\beta_{-}^{\prime 2}\right) / \rho \rightarrow 0$, that are consistent with our above remark. In the present case the comparison with the density should 
include the contribution of the scalar field. We define an anisotropic density $\rho_{a}$ that is proportional to the shear scalar:

$$
\rho_{a}=\beta_{+}^{\prime 2}+\beta_{-}^{\prime 2}
$$

and will compare it with $\rho_{\gamma}, \rho_{\phi}$, and $\Omega^{\prime 2}$. From the Hamilton Jacobi analysis we know that

$$
\begin{gathered}
\rho_{a} \sim e^{-6 \Omega}, \quad \rho_{\phi} \sim e^{-6 \Omega}, \\
\Omega^{\prime 2} \sim 48 \Lambda+\kappa_{\Omega}^{2} e^{-6 \Omega}+b_{\gamma} e^{-3(1+\gamma) \Omega}
\end{gathered}
$$

and the ratios are

$$
\begin{aligned}
& \frac{\rho_{a}}{\rho_{\phi}} \sim \text { constant, } \quad \frac{\rho_{a}}{\rho_{\gamma}} \sim e^{3 \Omega(\gamma-1)}, \\
& \frac{\rho_{a}}{\Omega^{\prime 2}} \sim \frac{1}{\kappa_{\Omega}^{2}+48 \Lambda e^{6 \Omega}+b_{\gamma} e^{3(1-\gamma) \Omega}} .
\end{aligned}
$$

Here we see that for expanding a universe the anisotropic density is dominated by the fluid density (with the exception of the stiff fluid) or by the $\Omega^{\prime 2}$ term and then at late times the isotropization is obtained if the expansion goes to infinity. Hence it is necessary to determine when we have an ever expanding universe. Equation (34) in the new variable $V=$ $e^{3 \Omega}$ is

$$
16 V^{\prime 2}-b_{\gamma} V^{1-\gamma}-48 \Lambda V^{2}=\kappa_{\Omega}{ }^{2}, \quad \kappa_{\Omega}{ }^{2}=\kappa_{+}{ }^{2}+\kappa_{-}{ }^{2}+\kappa_{\phi}{ }^{2} .
$$

That is equivalent to the equation of motion in the coordinate $V$ of a particle under the potential $U$ with energy $E=\kappa_{\Omega}^{2}$, where

$$
U(V)=-b_{\gamma} V^{1-\gamma}-48 \Lambda V^{2} .
$$

We can now have a qualitative idea of the different solutions from energy diagrams. We assume that $b_{\gamma}$ is nonnegative since it is proportional to the energy density of the fluid. On the other hand, $\Lambda$ and $\kappa_{\Omega}{ }^{2}$ are real and can take positive, null, or negative values.

From Figure 1 that is qualitatively correct for $\gamma \neq 1$, we see that for negative $\Lambda$ all the expanding solutions will recollapse eventually, regardless of the sign of $\kappa_{\Omega}^{2}$. For positive $\Lambda$ an expanding solution will expand forever. We also note that, when $\kappa_{\Omega}^{2}<0$, that is, when the ghost contribution is dominant, there are contracting solutions that reach a minimum and then expand forever; these solutions do not have a big bang singularity.

2.2.1. Radiation Case, $\mathscr{G}=X^{2}$. Reproducing the set of equation for this case, we have the fact that the Friedmann-like equation is

$$
3 \Omega^{\prime 2}-3 \beta_{+}^{\prime 2}-3 \beta_{-}^{\prime 2}-\frac{3}{4} \phi^{\prime 4}-\rho-\Lambda=0,
$$

and, making the same analysis that is in the previous case, we have

$$
\begin{array}{r}
3 \Omega^{\prime 2}=3 c_{1} e^{-6 \Omega}+3 \phi_{1} e^{-4 \Omega}+\mu_{\gamma} e^{-3(\gamma+1) \Omega}+\Lambda, \\
c_{1}=a_{+}^{2}+a_{-}^{2} .
\end{array}
$$

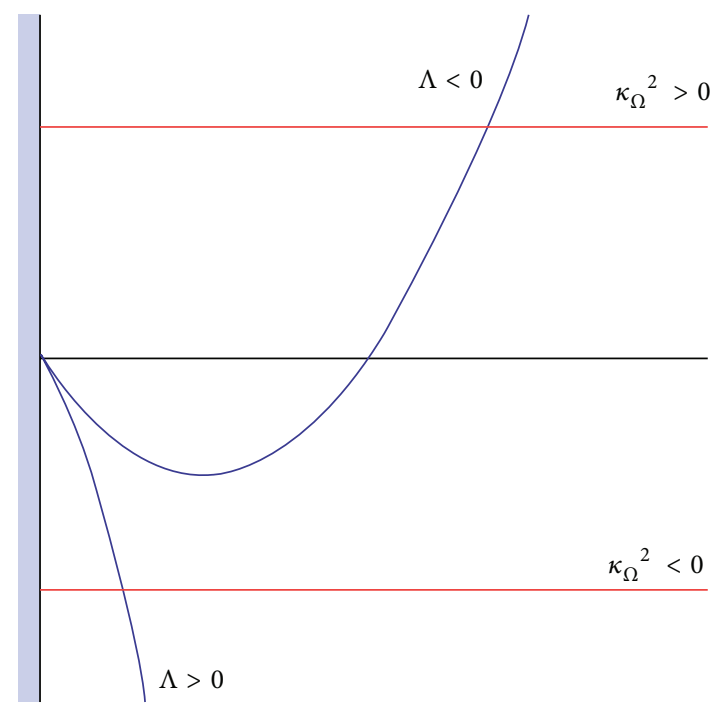

FIgURE 1: Plot of (39), considering different classes of total energy, in the $\kappa_{\Omega}^{2}$ parameter. Also, we include two branches in the cosmological constant.

Also the relation between the anisotropic function $\beta_{ \pm}$and (32) is satisfied. In the last equation we have used (14).

Also, we can follow the same structure for the matter case and, following the Hamilton Jacobi analysis, we know that

$$
\begin{gathered}
\rho_{a} \sim e^{-6 \Omega}, \quad \rho_{\phi} \sim e^{-4 \Omega}, \\
\Omega^{\prime 2} \sim 48 \Lambda+\phi_{1} e^{-4 \Omega}+\kappa_{\Omega}{ }^{2} e^{-6 \Omega}+b_{\gamma} e^{-3(1+\gamma) \Omega}
\end{gathered}
$$

and the corresponding ratios are

$$
\begin{gathered}
\frac{\rho_{a}}{\rho_{\phi}} \sim e^{-2 \Omega}, \quad \frac{\rho_{a}}{\rho_{\gamma}} \sim e^{3 \Omega(\gamma-1)}, \\
\frac{\rho_{a}}{\Omega^{\prime 2}} \sim \frac{1}{\kappa_{\Omega}^{2}+48 \Lambda e^{6 \Omega}+\phi_{1} e^{2 \Omega}+b_{\gamma} e^{3(1-\gamma) \Omega}} .
\end{gathered}
$$

Hence it is necessary to determine when we have an ever expanding universe. Equation (41) in the new variable $V=$ $e^{3 \Omega}$

$$
\begin{gathered}
16 V^{\prime 2}-b_{\gamma} V^{1-\gamma}-48 \Lambda V^{2}-c V^{2 / 3}=E_{\Omega}{ }^{2}, \\
E_{\Omega}{ }^{2}=\kappa_{+}{ }^{2}+\kappa_{-}{ }^{2} .
\end{gathered}
$$

That is equivalent to the equation of motion in the coordinate $V$ of a particle under the potential $U$ with energy $E=E_{\Omega}{ }^{2}$, where

$$
U(V)=-b_{\gamma} V^{1-\gamma}-48 \Lambda V^{2}-c V^{2 / 3} .
$$

For this case, the qualitative analysis is the same when $\mathscr{G}=X$.

In the following we obtain exact solutions in order to give the volume function $V$ to each case to $\mathscr{G}=X$.

2.3. Exact Classical Solutions. In order to find the solutions for the remaining minisuperspace variables we employ the Einstein-Hamilton-Jacobi equation, which arises by making 
the identification $\partial S\left(\Omega, \beta_{ \pm}, \phi\right) / \partial q^{\mu}=\Pi_{\mu}$ in the Hamiltonian constraint $\mathscr{H}_{\perp}=0$, which results in

$$
\begin{gathered}
\left(\frac{\partial S}{\partial \Omega}\right)^{2}-\left(\frac{\partial S}{\partial \beta_{+}}\right)^{2}-\left(\frac{\partial S}{\partial \beta_{-}}\right)^{2}+\frac{12}{f(\phi)}\left(\frac{\partial S}{\partial \phi}\right)^{2} \\
-b_{\gamma} e^{3 \Omega(1-\gamma)}-48 \Lambda e^{6 \Omega}=0
\end{gathered}
$$

In order to solve the above equation, we assume a solution of the form $S\left(\Omega, \beta_{+}, \phi\right)=S_{1}(\Omega)+S_{2}\left(\beta_{+}\right)+S_{3}\left(\beta_{-}\right)+S_{4}(\phi)$ which results in the following set of ordinary differential equations:

$$
\begin{gathered}
\left(\frac{d S_{1}}{d \Omega}\right)^{2}-\left(b_{\gamma} e^{-3(\gamma-1) \Omega}+48 \Lambda e^{6 \Omega}+\kappa_{\Omega}^{2}\right)=0, \\
\left(\frac{d S_{2}}{d \beta_{+}}\right)^{2}-\kappa_{+}{ }^{2}=0, \\
\left(\frac{d S_{3}}{d \beta_{-}}\right)^{2}-\kappa_{-}^{2}=0, \\
\frac{12}{f(\phi)}\left(\frac{d S_{4}}{d \phi}\right)^{2}-\kappa_{\phi}^{2}=0 .
\end{gathered}
$$

Here the $\kappa_{i}$ are separation constants satisfying the relation $\kappa_{\Omega}{ }^{2}=\kappa_{+}{ }^{2}+\kappa_{-}^{2}+\kappa_{\phi}^{2}, \kappa_{ \pm}$are real, $\kappa_{\phi}^{2}$ should have the same signs as $f(\phi)$, and for consistency with (19) we have $\kappa_{\phi}^{2}=24 \eta$. Recalling the expressions for the momenta we can obtain solutions for equations (47)-(48) in quadrature; in particular

$$
\begin{gathered}
\Delta \tau=12 \int \frac{d \Omega}{\sqrt{48 \Lambda+\kappa_{\Omega}^{2} e^{-6 \Omega}+b_{\gamma} e^{-3(1+\gamma) \Omega}}}, \\
\Delta \beta_{ \pm}=\mp \frac{\kappa_{ \pm}}{12} \int e^{-3 \Omega(\tau)} d \tau .
\end{gathered}
$$

We already know the solution for (48). As can be seen from (50), in order to obtain solutions for $\beta_{ \pm}$one needs to find a solution for $\Omega$, which can be obtained from (49).

Equation (49) does not have a general solution; however, it is possible to find solutions for particular values of the barotropic parameter $\gamma$ with $\Lambda \neq 0$.

(1) $\Lambda=0$ and $\gamma \neq 1$. Equation (49) can be written as

$$
d \tau=12 \frac{e^{3 \Omega} d \Omega}{\sqrt{\kappa_{\Omega}^{2}+b_{\gamma} e^{-3 \Omega(\gamma-1)}}} .
$$

When we consider the time transformations $d \tau=e^{3 \gamma \Omega} d T$, and the change of variable $u=\kappa_{\Omega}{ }^{2}+b_{\gamma} e^{-3(\gamma-1) \Omega}$, this equation has the solution

$$
\Omega(T)=\ln \left[\theta_{\gamma} T^{2}+\delta_{\gamma} T\right]^{-1 / 3(\gamma-1)},
$$

where $\theta_{\gamma}=((\gamma-1) / 8)^{2} b_{\gamma}$ and $\delta_{\gamma}=-\sqrt{\kappa_{\Omega}^{2}}((\gamma-1) / 4)$. With this, the time transformation becomes

$$
d \tau=\left[\theta_{\gamma} T^{2}+\delta_{\gamma} T\right]^{-\gamma /(\gamma-1)} d T .
$$

And the closed form is [22]

$$
\begin{aligned}
\tau= & \frac{(1-\gamma)}{\delta_{\gamma}}\left[\theta_{\gamma} T^{2}+\delta_{\gamma} T\right]^{1 /(1-\gamma)} \\
& \times{ }_{2} F_{1}\left(1,-\frac{2}{\gamma-1} ; \frac{\gamma-2}{\gamma-1} ;-\frac{T \theta_{\gamma}}{\delta_{\gamma}}\right),
\end{aligned}
$$

where ${ }_{2} F_{1}$ is a hypergeometric function. We also have

$$
\int e^{-3 \Omega} d \tau=\frac{1}{\delta_{\gamma}} \ln \left[\frac{T}{\theta_{\gamma} T+\delta_{\gamma}}\right] .
$$

The anisotropy functions and the scalar field are given by

$$
\begin{gathered}
\Delta \beta_{ \pm}=\mp \frac{\kappa_{ \pm}}{12 \delta_{\gamma}} \ln \left[\frac{T}{\theta_{\gamma} T+\delta_{\gamma}}\right], \\
{\left[(m+2) \sqrt{\frac{\eta}{2 \omega}} \frac{1}{\delta_{\gamma}} \ln \left[\frac{T}{\theta_{\gamma} T+\delta_{\gamma}}\right]\right]^{2 /(m+2)},} \\
f(\phi)=\omega \phi^{m}, \quad m \neq-2, \\
\operatorname{Exp}\left\{\sqrt{\left.\frac{2 \eta}{\omega} \frac{1}{\delta_{\gamma}} \ln \left[\frac{T}{\theta_{\gamma} T+\delta_{\gamma}}\right]\right\},}\right. \\
f(\phi)=\omega \phi^{-2}, \quad m=-2, \\
\frac{2}{m} \ln \left[m \sqrt{\frac{\eta}{2 \omega}} \frac{1}{\delta_{\gamma}} \ln \left[\frac{T}{\theta_{\gamma} T+\delta_{\gamma}}\right]\right], \\
f(\phi)=\omega e^{m \phi}, \quad m \neq 0, \\
\sqrt{2 \eta} \frac{1}{\delta_{\gamma}} \ln \left[\frac{T}{\theta_{\gamma} T+\delta_{\gamma}}\right], \\
f(\phi)=\omega, \quad m=0 .
\end{gathered}
$$

As a concrete example we consider the particular value $\gamma=0$ and then $\tau=T$ and $\Omega$ becomes

$$
\begin{aligned}
\Omega(\tau) & =\ln \left[\frac{3}{4} \mu_{0} T^{2}+\frac{\sqrt{\kappa_{\Omega}^{2}}}{4} T\right]^{1 / 3}, \\
& \Longrightarrow e^{3 \Omega}=\frac{3}{4} \mu_{0} T^{2}+\frac{\sqrt{\kappa_{\Omega}^{2}}}{4} T .
\end{aligned}
$$

If isotropization is possible, this volume function would not make it quick, and the integral becomes

$$
\int e^{-3 \Omega} d \tau=\frac{4}{\sqrt{\kappa_{\Omega}^{2}}} \ln \left[\frac{\tau}{\left(\sqrt{\kappa_{\Omega}^{2}} / 4\right)+(3 / 4) \mu_{0} \tau}\right] .
$$


So, the classical solutions for the anisotropic function $\beta_{ \pm}$and $\phi$ field are

$$
\begin{aligned}
& \Delta \beta_{ \pm}=\mp \frac{\kappa_{ \pm}}{3 \sqrt{\kappa_{\Omega}^{2}}} \ln \left[\frac{\tau}{\left(\sqrt{\kappa_{\Omega}^{2}} / 4\right)+(3 / 4) \mu_{0} \tau}\right], \\
& \int\left[(m+2) \sqrt{\frac{\eta}{2 \omega}} \ln \left[\frac{\tau}{\left(\sqrt{\kappa_{\Omega}^{2}} / 4\right)+(3 / 4) \mu_{0} \tau}\right]\right]^{2 /(m+2)} \text {, } \\
& f(\phi)=\omega \phi^{m}, \quad m \neq-2, \\
& \operatorname{Exp}\left\{\sqrt{\frac{2 \eta}{\omega}} \ln \left[\frac{\tau}{\left(\sqrt{\kappa_{\Omega}^{2}} / 4\right)+(3 / 4) \mu_{0} \tau}\right]\right\} \text {, } \\
& \phi(\tau)=\left\{\begin{array}{c}
f(\phi)=\omega \phi^{-2}, \quad m=-2, \\
\frac{2}{m} \ln \left[m \sqrt{\frac{\eta}{2 \omega}} \ln \left[\frac{\tau}{\left(\sqrt{\kappa_{\Omega}^{2}} / 4\right)+(3 / 4) \mu_{0} \tau}\right]\right],
\end{array}\right. \\
& f(\phi)=\omega e^{m \phi}, \quad m \neq 0, \\
& \sqrt{2 \eta} \frac{4}{\sqrt{\kappa_{\Omega}^{2}}} \ln \left[\frac{\tau}{\left(\sqrt{\kappa_{\Omega}^{2}} / 4\right)+(3 / 4) \mu_{0} \tau}\right] \text {, } \\
& f(\phi)=\omega, \quad m=0 \text {. }
\end{aligned}
$$

(2) $\Lambda=0$ and $\gamma=1$. In this case (49) is

$$
\Delta \tau=\int \frac{12}{\sqrt{b_{2} e^{-6 \Omega}}} d \Omega
$$

with $b_{2}=\kappa_{\Omega}{ }^{2}+48 \mu_{1}$ that we assume positive. By integrating we obtain

$$
e^{3 \Omega}=\frac{\sqrt{b_{2}} \Delta \tau}{4}
$$

If isotropization is possible, this volume function would not make it quick. For the anisotropic functions we have

$$
\Delta \beta_{ \pm}=\mp \frac{\kappa_{ \pm}}{12} \int e^{-3 \Omega(\tau)} d \tau=\mp \kappa_{ \pm} \frac{1}{3 \sqrt{b_{2}}} \ln (\Delta \tau),
$$

and the scalar field is given by

$$
\phi(\tau)=\left\{\begin{array}{c}
{\left[(m+2) \sqrt{\frac{\eta}{2 \omega}} \frac{4}{\sqrt{b_{2}}} \ln (\Delta \tau)\right]^{2 /(m+2)},} \\
f(\phi)=\omega \phi^{m}, \quad m \neq-2, \\
\operatorname{Exp}\left\{\sqrt{\frac{2 \eta}{\omega}} \frac{4}{\sqrt{b_{2}}} \ln (\Delta \tau)\right\}, \\
f(\phi)=\omega \phi^{-2}, \quad m=-2, \\
\frac{2}{m} \ln \left[m \sqrt{\frac{\eta}{2 \omega}} \frac{4}{\sqrt{b_{2}}} \ln (\Delta \tau)\right], \\
f(\phi)=\omega e^{m \phi}, \quad m \neq 0, \\
\sqrt{2 \eta} \frac{4}{\sqrt{b_{2}}} \ln (\Delta \tau), \quad \\
f(\phi)=\omega, \quad m=0 .
\end{array}\right.
$$

(3) $\Lambda \neq 0$ and $\gamma=-1$. Equation (49) has the form

$$
\Delta \tau=\int \frac{12}{\sqrt{\kappa_{\Omega}^{2} e^{-6 \Omega}+b_{3}}} d \Omega,
$$

where $b_{3}=48 \mu_{-1}+48 \Lambda$ :

$$
\Delta \tau=\frac{4}{\sqrt{b_{3}}} \operatorname{arccsch}\left(\sqrt{\frac{\kappa_{\Omega}^{2}}{b_{3}}} e^{-3 \Omega}\right) .
$$

Solving for $\Omega$

$$
\Omega=\frac{1}{3} \ln \left|\sqrt{\frac{\kappa_{\Omega}^{2}}{b_{3}}} \sinh \left(\frac{\sqrt{b_{3}}}{4} \Delta \tau\right)\right| .
$$

The inverse volume function is

$$
e^{-3 \Omega}=\sqrt{\frac{b_{3}}{\kappa_{\Omega}^{2}}} \operatorname{csch}\left(\frac{\sqrt{b_{3}}}{4} \Delta \tau\right) .
$$

If isotropization is possible, the corresponding volume function would make it quick and its integral becomes

$$
\int e^{-3 \Omega} d \tau=\frac{4}{\sqrt{\kappa_{\Omega}^{2}}} \ln \left|\tanh \left(\frac{\sqrt{b_{3}}}{4} \Delta \tau\right)\right| .
$$

And, as the anisotropic function is dependent on this integral, so

$$
\Delta \beta_{ \pm}(\tau)= \pm \frac{\kappa_{ \pm}}{3 \sqrt{\kappa_{\Omega}^{2}}} \ln \left|\tanh \left(\frac{\sqrt{b_{3}}}{4} \Delta \tau\right)\right| .
$$

Also, the field $\phi$ uses this integral (see (20)), and the corresponding solutions become

$$
\phi(\tau)=\left\{\begin{array}{c}
{\left[-(m+2) \sqrt{\frac{\eta}{2 \omega}} \frac{4}{\sqrt{\kappa_{\Omega}^{2}}} \ln \left|\tanh \left(\frac{\sqrt{b_{3}}}{4} \Delta \tau\right)\right|\right]^{2 /(m+2)},} \\
f(\phi)=\omega \phi^{m}, \quad m \neq-2, \\
\operatorname{Exp}\left\{-\sqrt{\frac{2 \eta}{\omega}} \frac{4}{\sqrt{\kappa_{\Omega}^{2}}} \ln \left|\tanh \left(\frac{\sqrt{b_{3}}}{4} \Delta \tau\right)\right|\right\} \\
f(\phi)=\omega \phi^{-2}, \quad m=-2, \\
\frac{2}{m} \ln \left[-m \sqrt{\frac{\eta}{2 \omega}} \frac{4}{\sqrt{\kappa_{\Omega}^{2}}} \ln \left|\tanh \left(\frac{\sqrt{b_{3}}}{4} \Delta \tau\right)\right|\right] \\
f(\phi)=\omega e^{m \phi}, \quad m \neq 0, \\
-\sqrt{2 \eta} \frac{4}{\sqrt{\kappa_{\Omega}^{2}}} \ln \left|\tanh \left(\frac{\sqrt{b_{3}}}{4} \Delta \tau\right)\right| \\
f(\phi)=\omega, \quad m=0
\end{array}\right.
$$

with the condition $\omega>0$.

Also we can consider the special case, when $b_{3}=0$ or $\mu_{-1}=-\Lambda$ :

$$
\begin{gathered}
\Delta \tau=\frac{4}{\sqrt{\kappa_{\Omega}^{2}}} e^{3 \Omega}, \\
\Omega=\frac{1}{3} \ln \left|\frac{\sqrt{\kappa_{\Omega}^{2}}}{4} \Delta \tau\right| .
\end{gathered}
$$


We have the following expression:

$$
e^{-3 \Omega}=\frac{4}{\sqrt{\kappa_{\Omega}^{2}}} \frac{1}{\Delta \tau}
$$

The isotropization is possible for this case, because the corresponding volume function would make it quick and its integral becomes

$$
\int e^{-3 \Omega} d \tau=\frac{4}{\sqrt{\kappa_{\Omega}^{2}}} \ln |\Delta \tau|
$$

So, the anisotropic function $\beta_{ \pm}$and the $\phi$ function become for this case

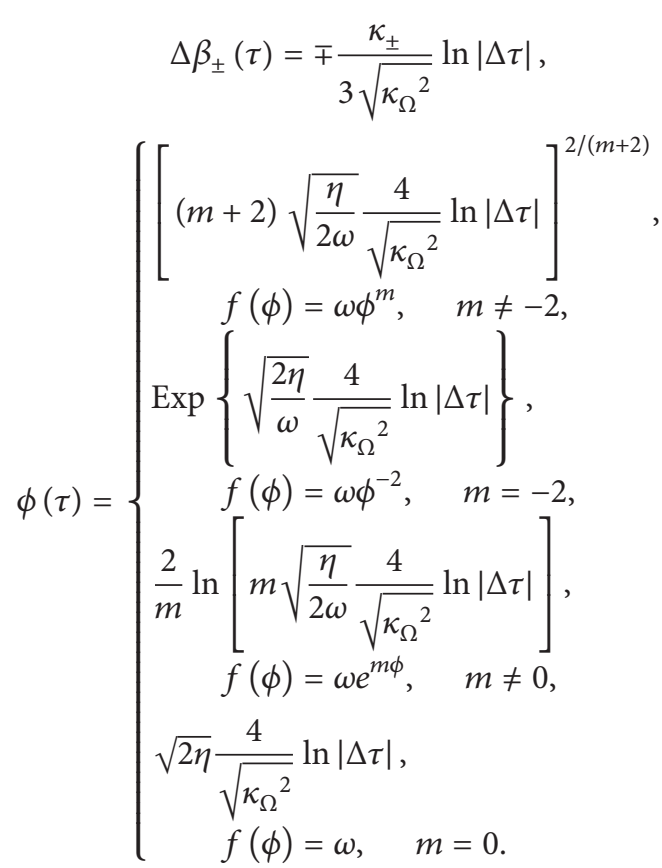

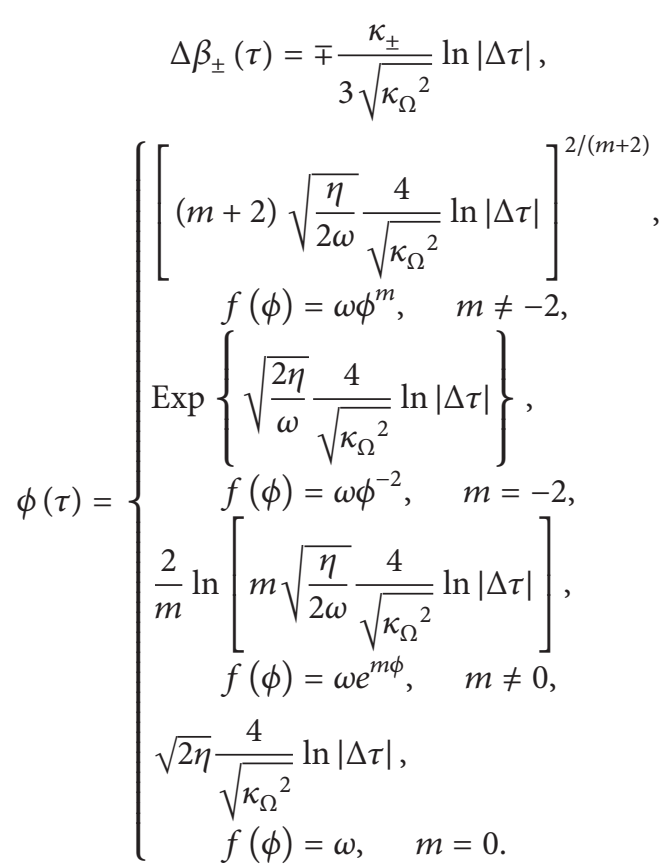

(4) $\Lambda \neq 0$ and $\gamma=0$. For this case, (49) is

$$
\begin{aligned}
\Delta \tau= & \int \frac{12 e^{3 \Omega}}{\sqrt{\kappa_{\Omega}^{2}+48 \mu_{0} e^{3 \Omega}+48 \Lambda e^{6 \Omega}}} d \Omega \\
= & \frac{1}{\sqrt{3 \Lambda}} \\
& \times \ln \left[\frac{b_{0}+96 \Lambda e^{3 \Omega}}{48 \Lambda}+\frac{1}{2 \sqrt{3 \Lambda}} \sqrt{\kappa_{\Omega}^{2}+b_{0} e^{3 \Omega}+48 \Lambda e^{6 \Omega}}\right]
\end{aligned}
$$

with $b_{0}=48 \mu_{0}$ and $\Lambda>0$.

The function $\Omega$ becomes

$$
\Omega=\frac{1}{3} \ln \left[\frac{12 \Lambda\left(e^{\sqrt{3 \Lambda} \Delta \tau}-\left(b_{0} / 48 \Lambda\right)\right)^{2}-\kappa_{\Omega}^{2}}{48 \Lambda e^{\sqrt{3 \Lambda} \Delta \tau}}\right]
$$

and we have

$$
\begin{gathered}
e^{-3 \Omega}=\frac{48 \Lambda e^{\sqrt{3 \Lambda} \Delta \tau}}{12 \Lambda\left(e^{\sqrt{3 \Lambda} \Delta \tau}-\left(b_{0} / 48 \Lambda\right)\right)^{2}-\kappa_{\Omega}{ }^{2}} \\
\int e^{-3 \Omega} d \tau=-\frac{8}{\sqrt{\kappa_{\Omega}^{2}}} \operatorname{arctanh}\left(2 \sqrt{\frac{3 \Lambda}{\kappa_{\Omega}^{2}}}\left(-\frac{b_{0}}{48 \Lambda}+e^{\sqrt{3 \Lambda} \Delta \tau}\right)\right) .
\end{gathered}
$$

Also, the isotropization is possible, because the corresponding volume function would make it quick. For the anisotropic function $\beta_{ \pm}$we have

$$
\Delta \beta_{ \pm}(\tau)= \pm \frac{2 \kappa_{ \pm}}{3 \sqrt{\kappa_{\Omega}^{2}}} \operatorname{arctanh}\left(2 \sqrt{\frac{3 \Lambda}{\kappa_{\Omega}^{2}}}\left(-\frac{b_{0}}{48 \Lambda}+e^{\sqrt{3 \Lambda} \Delta \tau}\right)\right)
$$

and for the $\phi$ function

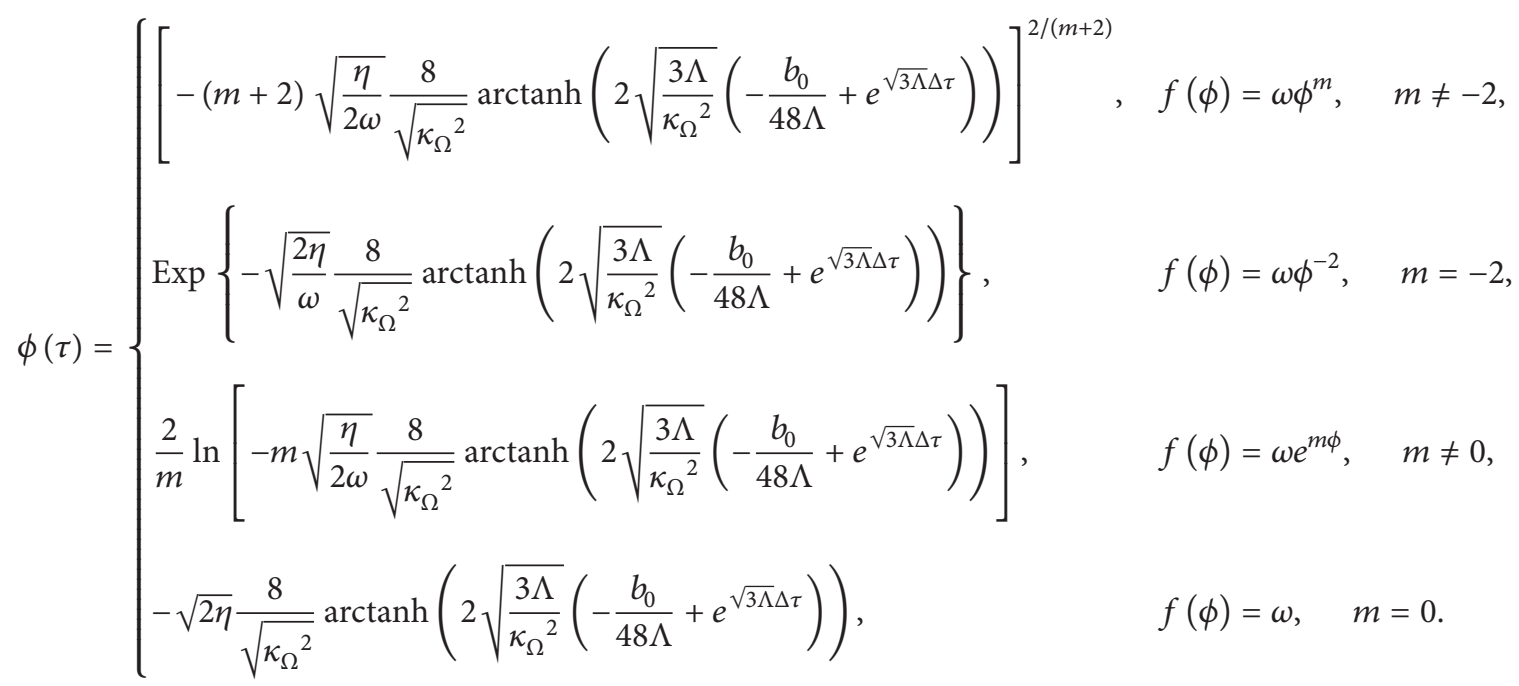


(5) $\Lambda \neq 0$ and $\gamma=1$. Equation (49) becomes

$$
\Delta \tau=\int \frac{12}{\sqrt{b_{4} e^{-6 \Omega}+48 \Lambda}} d \Omega,
$$

where $b_{4}=\kappa_{\Omega}{ }^{2}+48 \mu_{1}$. In this case also we have two possible solutions depending on the value of the cosmological constant.

(i) $\Lambda>0$. The solution becomes

$$
\Delta \tau=\frac{1}{\sqrt{3 \Lambda}} \operatorname{arcsinh}\left(4 \sqrt{\frac{3 \Lambda}{b_{4}}} e^{3 \Omega}\right)
$$

so the function $\Omega$ is

$$
\begin{gathered}
\Omega=\frac{1}{3} \ln \left|\frac{1}{4} \sqrt{\frac{b_{4}}{3 \Lambda}} \sinh (\sqrt{3 \Lambda} \Delta \tau)\right|, \\
e^{-3 \Omega}=4 \sqrt{\frac{3 \Lambda}{b_{4}}} \operatorname{csch}(\sqrt{3 \Lambda} \Delta \tau), \\
\int e^{-3 \Omega} d \tau=\frac{4}{\sqrt{b_{4}}} \ln [\tanh (\sqrt{3 \Lambda} \Delta \tau)] .
\end{gathered}
$$

For this case the isotropization is possible and the corresponding volume function would make it quick. For the anisotropic function $\beta_{ \pm}$we have

$$
\Delta \beta_{ \pm}= \pm \frac{\kappa_{ \pm}}{3 \sqrt{b_{4}}} \ln [\tanh (\sqrt{3 \Lambda} \Delta \tau)]
$$

and for the $\phi$ field

$$
\phi(\tau)=\left\{\begin{array}{c}
{\left[-(m+2) \sqrt{\frac{\eta}{2 \omega}} \frac{4}{\sqrt{b_{4}}} \ln [\tanh (\sqrt{3 \Lambda} \Delta \tau)]\right]^{2 /(m+2)},} \\
f(\phi)=\omega \phi^{m}, \quad m \neq-2, \\
\operatorname{Exp}\left\{-\sqrt{\frac{2 \eta}{\omega}} \frac{4}{\sqrt{b_{4}}} \ln [\tanh (\sqrt{3 \Lambda} \Delta \tau)]\right\}, \\
f(\phi)=\omega \phi^{-2}, \quad m=-2, \\
\frac{2}{m} \ln \left[-m \sqrt{\frac{\omega \eta}{2 \omega}} \frac{4}{\sqrt{b_{4}}} \ln [\tanh (\sqrt{3 \Lambda} \Delta \tau)]\right], \\
f(\phi)=\omega e^{m \phi}, \quad m \neq 0, \\
-\sqrt{2 \eta} \frac{4}{\sqrt{b_{4}}} \ln [\tanh (\sqrt{3 \Lambda} \Delta \tau)], \\
f(\phi)=\omega, \quad m=0 .
\end{array}\right.
$$

(ii) $\Lambda<0$. The corresponding solutions are

$$
\Delta \tau=-\frac{1}{\sqrt{3|\Lambda|}} \arccos \left(4 \sqrt{\frac{3|\Lambda|}{b_{4}}} e^{3 \Omega}\right) .
$$

The function $\Omega$ is

$$
\Omega=\frac{1}{3} \ln \left|\frac{1}{4} \sqrt{\frac{b_{4}}{3|\Lambda|}} \cos (\sqrt{3|\Lambda|} \Delta \tau)\right| .
$$

As the volume has an oscillatory behavior, the isotropization does not yield for this case, and for completeness we calculate

$$
\begin{gathered}
e^{-3 \Omega}=4 \sqrt{\frac{3|\Lambda|}{b_{4}}} \sec (\sqrt{3|\Lambda|} \Delta \tau), \\
\int e^{-3 \Omega} d \tau=\frac{4}{\sqrt{b_{4}}} \ln |\sec (\sqrt{3|\Lambda|} \Delta \tau)+\tan (\sqrt{3|\Lambda|} \Delta \tau)| \\
=\frac{4}{\sqrt{b_{4}}} \ln \left|\frac{\cos ((\sqrt{3|\Lambda|} / 2) \Delta \tau)+\sin ((\sqrt{3|\Lambda|} / 2) \Delta \tau)}{\cos ((\sqrt{3|\Lambda|} / 2) \Delta \tau)-\sin (\sqrt{3|\Lambda|} / 2)}\right| .
\end{gathered}
$$

The anisotropic function $\beta_{ \pm}$,

$$
\Delta \beta_{ \pm}=\mp \frac{\kappa_{ \pm}}{3 \sqrt{b_{4}}} \ln |\sec (\sqrt{3|\Lambda|} \Delta \tau)+\tan (\sqrt{3|\Lambda|} \Delta \tau)| .
$$

And the $\phi$ field

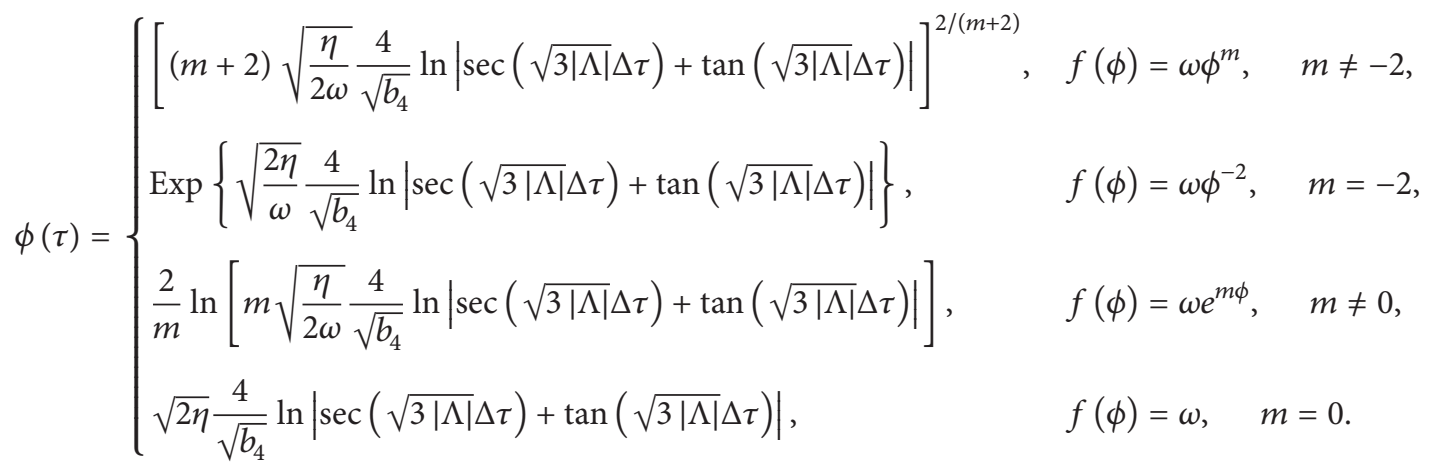




\section{Final Remarks}

In this work we present the study of the classical cosmological anisotropic Bianchi type I in the K-essence formalism. In previous work made by Chimento and Forte [16], they present the possible isotropization of this model. Our goal in this work is that we obtain the corresponding classical solutions for a barotropic perfect fluid and cosmological term $\Lambda$ which mimics the scalar field in (1). In the case of $\Lambda=0$ and $\gamma \neq 1$ we obtain the solutions in closed form. With these solutions we can validate our qualitative analysis on isotropization of the cosmological model, implying that this occurs when the volume is large in the corresponding time evolution. So, only one solution does not present the large volume, when $\Lambda<0$ in stiff matter era in the ordinary matter content. We include a qualitative analysis to Friedmann equation when it is written as an equation for the volume that is equivalent to the equation of motion of a particle under a potential and we conclude the same about the isotropization of this anisotropic model, considering the stiff matter and radiation cases. In [17] the author shows from the kinematic point of view the model when $G(X)$ is linear (as in our case) and the particular value to the function $f(\phi)$, using our notation, and the quintessence scalar field one driven by an exponential potential are the same, but they are dynamically nonequivalent because the K-field and the scalar potential are linked by the Einstein equation. However our results contain other functions $f(\phi)$; then we argued that explanation to the corresponding observational data and the kinematic properties, since each formalism will have big differences. All of these comments occur because the complete solution to the quintessence scalar field $\phi$ depends strongly on the behavior of the scale factor of the cosmological model under consideration and particular form to the function $f(\phi)$; Bianchi type model appears mimetic in the $\Omega$ function, as in (15) and (19), except when one chooses a particular gauge which includes this function, as in (21). In the quantum analysis for this model, considering the scalar field, the solutions are similar to those found in the Bianchi type IX cosmological model [23]; you can see (32). For quantum radiation case, the resulting Wheeler-DeWitt equation appears as fractionary differential equation, and the results will be reported elsewhere.

\section{Conflict of Interests}

The authors declare that there is no conflict of interests regarding the publication of this paper.

\section{Acknowledgments}

This work was partially supported by CONACYT Grants 167335 and 179881 and PROMEP Grants UGTO-CA-3 and UAM-I-43. This work is part of the collaboration within the Instituto Avanzado de Cosmología and Red PROMEP: Gravitation and Mathematical Physics under project Quantum Aspects of Gravity in Cosmological Models, Phenomenology, and Geometry of Space-Time. Many calculations were done by Symbolic Program REDUCE 3.8.

\section{References}

[1] D. Saez and V. J. Ballester, "A simple coupling with cosmological implications," Physics Letters A, vol. 113, no. 9, pp. 467-470, 1986.

[2] C. Armendariz-Picon, V. Mukhanov, and P. J. Steinhardt, "Dynamical solution to the problem of a small cosmological constant and late-time cosmic acceleration," Physical Review Letters, vol. 85, no. 21, pp. 4438-4441, 2000.

[3] C. Armendariz-Picon, V. Mukhanov, and P. J. Steinbardt, "Essentials of $k$-essence," Physical Review D, vol. 63, Article ID 103510, 2001.

[4] N. Bose and A. S. Majumdar, "K-essence model of inflation, dark matter, and dark energy," Physical Review D, vol. 79, no. 10, Article ID 103517, 2009.

[5] J. De-Santiago and J. L. Cervantes-Cota, "Generalizing a unified model of dark matter, dark energy, and inflation with non canonical kinetic term," Physical Review D, vol. 83, Article ID 063502, 2011.

[6] J. de-Santiago, J. L. Cervantes-Cota, and D. Wands, "Cosmological phase space analysis of the $F(X)-V(\varphi)$ scalar field and bouncing solutions," Physical Review D: Particles, Fields, Gravitation and Cosmology, vol. 87, no. 2, Article ID 023502, 2013.

[7] R. de Putter and E. V. Linder, "Kinetic k-essence and quintessence," Astroparticle Physics, vol. 28, no. 3, pp. 263-272, 2007.

[8] T. Chiba, S. Dutta, and R. J. Scherrer, "Slow-roll k-essence," Physical Review D: Particles, Fields, Gravitation and Cosmology, vol. 80, no. 4, Article ID 043517, 2009.

[9] F. Arroja and M. Sasaki, "A note on the equivalence of a barotropic perfect fluid with a k-essence scalar field," Physical Review D, vol. 81, Article ID 107301, 2010.

[10] L. A. García, J. M. Tejeiro, and L. Castañeda, "K-essence scalar field as dynamical dark energy," http://arxiv.org/abs/1210.5259.

[11] N. Bilic, G. Tupper, and R. Viollier, "Unification of dark matter and dark energy: the inhomogeneous Chaplygin gas," Physics Letters B, vol. 535, pp. 17-21, 2002.

[12] M. Bento, O. Bertolami, and A. Sen, "Generalized Chaplygin gas, accelerated expansion, and dark-energy-matter unification," Physical Review D, vol. 66, Article ID 043507, 2002.

[13] C. Armendariz-Picon, T. Damour, and V. Mukhanov, " $k$ Inflation," Physics Letters B, vol. 458, no. 2-3, pp. 209-218, 1999.

[14] J. Garriga and V. F. Mukhanov, "Perturbations in $k$-inflation," Physics Letters B, vol. 458, no. 2-3, pp. 219-225, 1999.

[15] E. J. Copeland, M. Sami, and S. Tsujikawa, "Dynamics of dark energy," International Journal of Modern Physics D, vol. 15, no. 11, article 1753, 2006.

[16] L. P. Chimento and M. Forte, "Anisotropic k-essence cosmologies," Physical Review D, vol. 73, no. 6, Article ID 063502, 2006.

[17] L. P. Chimento, "Extended tachyon field, Chaplygin gas, and solvable $k$-essence cosmologies," Physical Review D, vol. 69, no. 12, Article ID 123517, 10 pages, 2004.

[18] M. S. Madsen, "Scalar fields in curved spacetimes," Classical and Quantum Gravity, vol. 5, no. 4, pp. 627-639, 1988.

[19] L. O. Pimentel, "Energy-momentum tensor in the general scalar-tensor theory," Classical and Quantum Gravity, vol. 6, no. 12, pp. L263-L265, 1989.

[20] M. P. Ryan, Hamiltonian Cosmology, Springer, Berlin, Germany, 1972.

[21] E. Martinez-Gonzalez and J. L. Sanz, " $\Delta T / T$ and the isotropy of the universe," Astronomy \& Astrophysics, vol. 300, p. 346, 1995. 
[22] A. C. Polyanin and V. F. Zaitsev, Handbook of Exact Solutions for Ordinary Differential Equations, Chapman \& Hall/CRC, Boca Raton, Fla, USA, 2003.

[23] A. Espinoza-García, J. Socorro, and O. Luis, "Quantum bianchi type IX cosmology in K-essence theory," International Journal of Theoretical Physics, vol. 53, no. 9, pp. 3066-3077, 2014. 

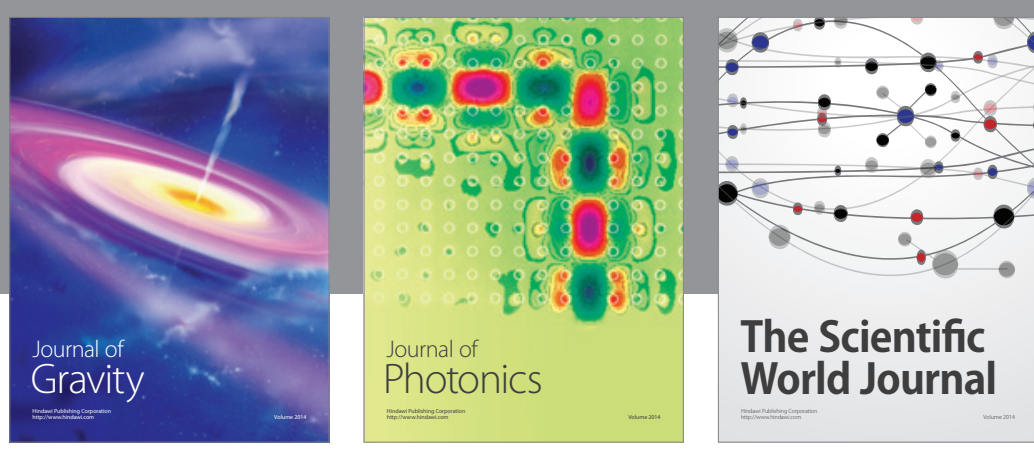

The Scientific World Journal
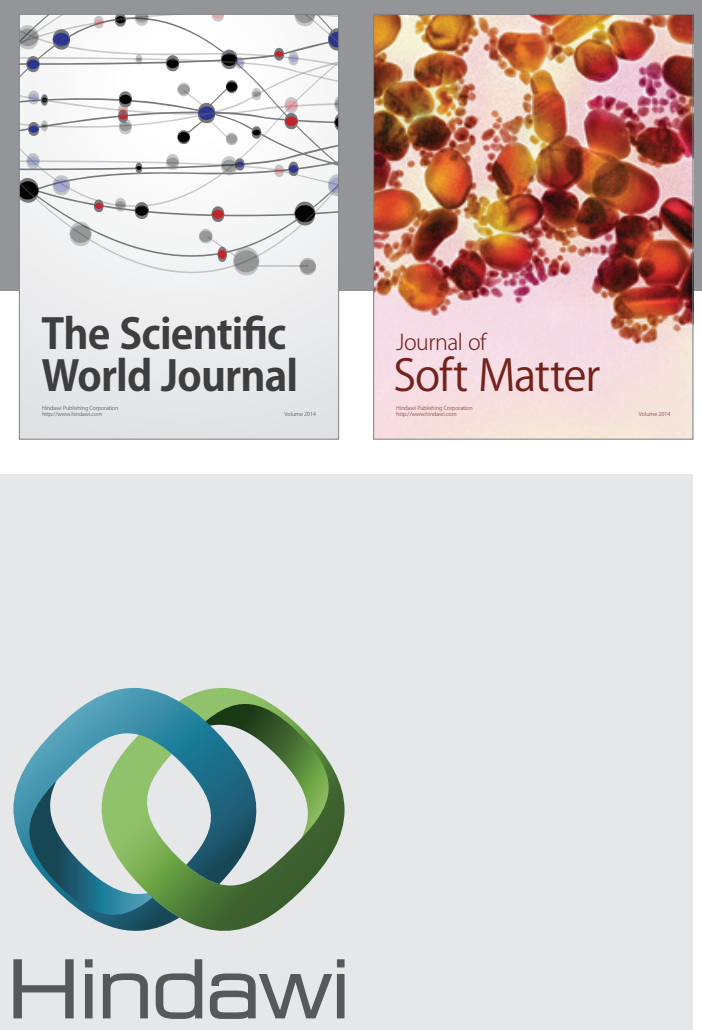

Submit your manuscripts at

http://www.hindawi.com

nternational Journal of

Statistical Mechanics
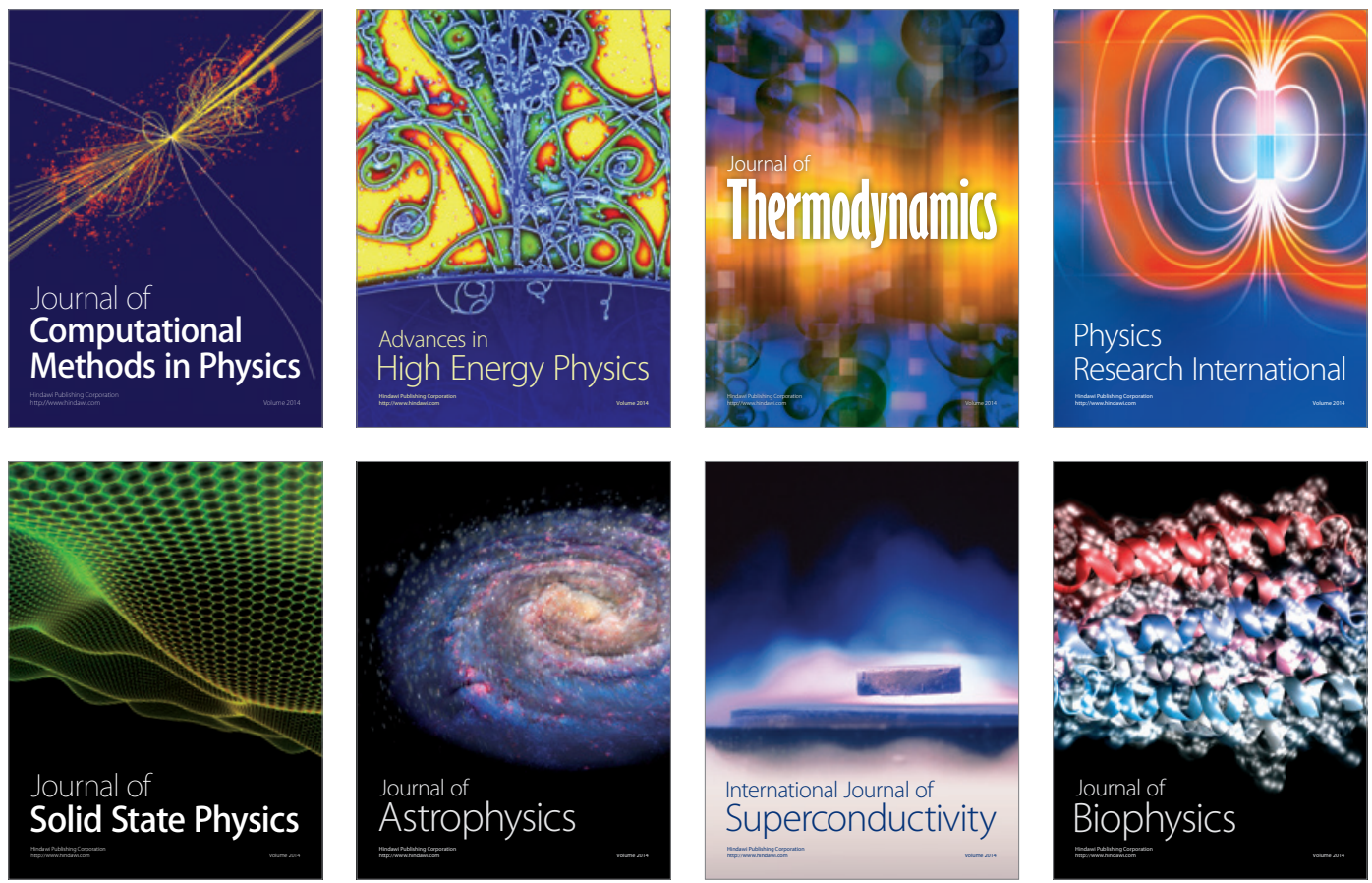
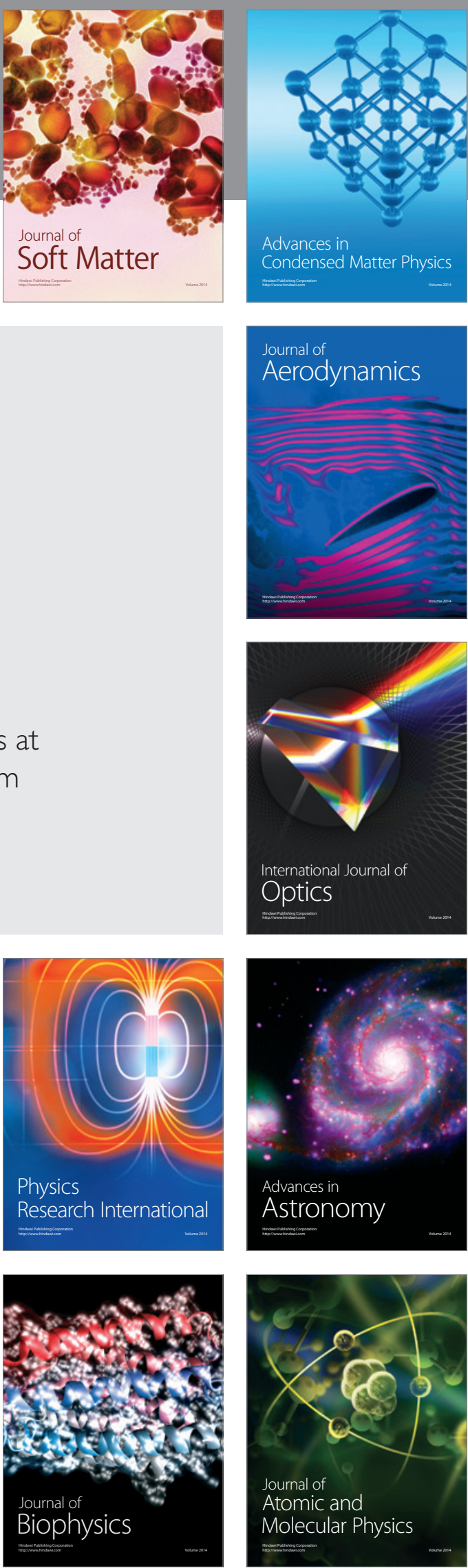